\title{
La importancia de la técnica de la entrevista en la investigación en comunicación y las ciencias sociales. Investigación documental. Ventajas y limitaciones
}

Pablo Fernández Juárez

https://doi.org/10.36105/stx.2018n1.07

\section{Resumen}

En el presente artículo se analiza la importancia de la técnica de entrevista dentro de la investigación cualitativa y documental en las ciencias sociales en general y en la comunicación y el periodismo en particular. Para ello se aborda el origen de la entrevista; asimismo, se explican tres tipos de entrevista (estructurada, no estructurada y de grupos), se analizan sus alcances y limitaciones, nuevas tendencias, así como los retos en el uso y abuso de la entrevista y se finaliza con las conclusiones del artículo.

Para esta investigación de naturaleza documental, de tipo argumentativa y exploratoria, se recopilaron y analizaron documentos bibliográficos, hemerográficos, videográficos y artículos de internet que se relacionaron con el tema en cuestión. Empezando con libros escritos sobre los orígenes de la técnica de entrevista psicológica, periodística y de investigación en ciencias sociales, así como libros, artículos y videos acerca de entrevistas diversas y de comentarios diversos acerca de dicha técnica.

\section{Palabras clave}

Entrevista, psicología, investigación cualitativa, investigación documental, tendencias.

1 Profesor de la Facultad de Comunicación e Investigador del Centro de Investigación para la Comunicación Aplicada (CICA) de la Universidad Anáhuac México.pfernan@anahuac.mx 


\section{¿Por qué es importante este tema?}

Para López y Deslauries (2011), "la entrevista tiene un enorme potencial que permite acceder a una parte vital de las personas a través de la cual descubrimos su cotidianidad y las relaciones sociales que mantienen" (p. 1). Es por esto que se posiciona como una técnica y herramienta fundamental para complementar el proceso cuantitativo de la investigación en las ciencias sociales.

Asimismo, estos autores sostienen que el método de encuesta (a través del cuestionario) tiene sus desventajas naturales, ya que no logra profundizar "en el fenómeno sociológico" ni en aspectos relevantes acerca de las actitudes, creencias y convicciones de los sujetos investigados, lo que sí permite la entrevista en sus diversas modalidades, ya sea de perfil, de profundidad, abierta, estructurada y no estructurada, entre otras" (Visauta, 1989, p. 259, citado en Hernández et al., 2015).

Por otra parte, es necesario señalar que esta técnica no es única e infalible a los errores propios del investigador y que, por su naturaleza misma, en ocasiones resulta ambigua y subjetiva en cuanto a las apreciaciones y percepción del entrevistador, independientemente de su entrenamiento, objetivo y experiencia.

Es importante señalar que, aunque algunos autores colocan a la encuesta como un método que a su vez incluye al cuestionario y a la entrevista como sus principales instrumento de recolección de datos, se puede clasificar esta última como una técnica que requiere una preparación y experiencia particulares y que puede demandar más recursos y tiempo de entrenamiento, a diferencia de un buen cuestionario; por lo mismo, la entrevista no es tan popular en las encuestas y en la investigación en ciencias sociales en general.

Los especialistas en investigación coinciden en recomendar su uso como complemento y en combinación con la observación sistemática y la misma encuesta, ya sea a través de cuestionarios y otros instrumentos auxiliares en la obtención e información, como los autorreportes, la autobiografía, y en su momento los tests proyectivos y de completamiento de frases (Anastasi, 1998).

Sin embargo, sus ventajas logran tener más peso e importancia si se siguen las reglas básicas en su aplicación, como registrar adecuadamente, con suficiente atención, objetividad $\mathrm{y}$ sin prejuicios las sutilezas del lenguaje no verbal, las reflexiones, gestos y actitudes que acompañan a las verbalizaciones del entrevistado, logrando con ello recolectar información más cercana a los pensamientos y actitudes y que permitan obtener confiabilidad y validez de las respuestas y de la propia metodología utilizada en general.

Finalmente, para Laura Díaz, Uri Torruco, Mildred Martínez y Margarita Varela (2013), del Departamento de Investigación de la Facultad de Medicina de la Universidad 
Nacional Autónoma de México, "La entrevista es muy ventajosa principalmente en los estudios descriptivos y en las fases de exploración, así como para diseñar instrumentos de recolección de datos”. (p. 3)

\section{Introducción}

En la investigación cualitativa de las ciencias sociales existen diferentes técnicas de colecta de datos cuyo propósito principal es obtener información de los participantes fundamentada en las percepciones, creencias, opiniones, significados y actitudes, por lo que la entrevista es una valiosa técnica que se estudiará en este análisis. En el presente artículo se realizará un recorrido teórico, con la aclaración de que la investigación está envuelta en una representación compleja de políticas, las cuales se ven reflejadas en la investigación social a través de las interacciones y las prácticas que se llevan a cabo, en donde el objeto de indagación constituye el principal aspecto de la investigación.

Para Denzin y Lincoln (2005), la entrevista es "una conversación, es el arte de realizar preguntas y escuchar respuestas” (p. 643). Además, esta técnica está fuertemente influenciada por las características personales del entrevistador; asimismo, ha llegado a convertirse en una actividad de nuestra cultura de masas, aunque la entrevista es un texto negociado, donde el poder, el género, la raza y los intereses de clases han sido de especial interés en los últimos tiempos.

Se inicia con los orígenes de la entrevista con el propósito de que los lectores conozcan la evolución de ésta en el campo de las ciencias sociales hasta el presente, se argumenta la entrevista desde la investigación cualitativa, se explican los tipos de entrevista (la estructurada, la no estructura y la grupal), la tipología de grupos de discusión elaborada por Fontana y Frey (2005), y se mencionan algunas nuevas tendencias de entrevista.

\section{Planteamiento del problema}

La técnica de entrevista en las ciencias humanas y sociales, y en particular en la comunicación, ha sufrido cambios y vaivenes tanto positivos como negativos.

En el primer caso, la depuración de la técnica y su metodología se han enriquecido a partir de las experiencias y evolución tanto en el ámbito clínico como en el periodístico.

Hay testimonios de grandes teóricos, como el psiquiatra Harry Stack Sullivan, creador del primer libro sobre el tema: La entrevista psiquiátrica, de 1948, y de famosos entrevista- 
dores como la periodista italiana Oriana Fallaci, quien en su texto de 1974, Entrevista con la bistoria, da cuenta de la técnica y el arte de la entrevista en sus respectivas modalidades y objetivos. Sin embargo, también hay periodistas, comunicadores y profesionales de la clínica que, sin preparación ni experiencia adecuada, se involucran en esta actividad de manera intuitiva, convencidos de que la espontaneidad y la informalidad son la base de una buena entrevista.

Se suman a lo anterior los entrevistadores que, guiados por propósitos ya determinados, dirigen al invitado por un camino ya planeado que transita desde preguntas que inicialmente sugieren una posible respuesta, hasta cuestionamientos y comentarios provocadores con el propósito de incomodarlo.

Es común escuchar -sobre todo en el ámbito periodístico- que una buena entrevista es aquella que obtiene información que merece ser publicada y que representa una valiosa noticia del momento, del hecho y de la vida misma del entrevistado bajo la premisa de que $e l$ público merece conocer la verdad a toda costa.

Por lo mismo, se plantea la tarea de recopilar información suficiente sobre la técnica misma, las diversas concepciones, definiciones y modalidades de ésta, incluyendo ejemplos que ilustren las tendencias, estilos y posibles fallas y aciertos en esta técnica de recolección de información.

\section{Conceptos básicos}

\section{Origen de la entrevista}

Se concibe a la entrevista como una técnica utilizada desde tiempos milenarios; según Fontana y Frey (2005), el uso de la entrevista se inicia desde la época ancestral egipcia cuando se conducían censos poblacionales. Asimismo, se destaca que es a partir del siglo XIX que la tradición de la entrevista evoluciona como técnica desde dos tendencias, la primera cuando la entrevista adquirió gran popularidad y expansión en casos de uso para diagnóstico clínico y orientación, y el segundo fue durante la Primera Guerra Mundial, donde la entrevista llegó a ser utilizada para pruebas de psicología con énfasis en medición (Maccoby \& Maccoby, citados en Fontana \& Frey, 2005).

Sin embargo, Lucca y Berríos (2003) señalan que no es sino a finales del siglo XIX cuando se comenzó a documentar el uso de la estrategia de la entrevista en la recopilación de información para usos de investigación, y mencionan como dato interesante al sociólogo Charles Booth, quien en 1886 realizó una encuesta social basada en entrevista. Según Denzin y Lincoln (2005), es a partir de la Primera Guerra Mundial que las encuestas de opinión 
ganaron terreno y se desarrollaron en los Estados Unidos, donde años después se pueden destacar los miembros de la Escuela de Chicago, alrededor de los años 1930, quienes utilizaron una combinación de observación, documentos personales y entrevistas formales en la realización de estudios (Vargas, 2011).

No obstante, en el caso de la entrevista periodística, Cristopher Silvester (1997) en su libro Las grandes entrevistas de la bistoria

asegura que la primera entrevista moderna, tal y como hoy la concebimos, fue publicada en el New York Tribune el 20 de agosto de 1859. Se trataba de una conversación con Bringham Young, un líder mormón, y fue realizada por Horace Greeley, un célebre periodista norteamericano (p. 27).

Es muy probable que en el centro de Europa se hayan realizado varias entrevistas desde el siglo XVII, cuando se empezaron a publicar los primeros periódicos.

Montero (1965, citado en Silvester, 1997) afirma que algunas de estas primeras entrevistas no tenían prestigio, ya que se centraban en aspectos frívolos, superficiales e incluso morbosos y amarillistas, por lo que no eran consideradas de importancia periodística ni mucho menos de investigación, esto a mediados del siglo XIX, en el auge del método científico.

De manera contradictoria, al ser considerada como vulgar y demasiado popular, en el siglo XX tuvo un éxito inusitado entre periodistas y escritores que llegaron a ser más conocidos por sus entrevistas que por su obra periodística o literaria. Tal es el caso, en la década de los cincuenta, de Truman Capote y su ya célebre novela $A$ sangre fría, que documenta de forma magistral tanto la técnica de la entrevista como el contexto dramático, caracterizado por un realismo frío y descarnado a través de un asesinato múltiple en el sur del estado de Kansas en 1959.

Así, a partir de 1965 se marca una distinción más clara entre la entrevista y el cuestionario, y es cuando se desarrolla una clasificación en diversas modalidades, según su conducción, formato y tipo de preguntas, como por ejemplo, la entrevista estructurada, que se utiliza como una estrategia apropiada para revelar información, en la que el entrevistador mantiene el control de la misma y lleva al entrevistado a contestar lo que estaba delimitado en los objetivos. Para principios de la década de los ochenta, se comienza a dar énfasis a la estrategia de la entrevista a profundidad, en donde el papel del entrevistador no sólo es obtener información, sino aprender qué tipo de pregunta se formula y cómo (Lucca \& Berríos, 2003).

Los etnógrafos posmodernos han considerado en años más recientes algunos supuestos en la entrevista con respecto a la función directiva y de guía del entrevistador, lo que según 
Fontana y Frey (2005) lleva a replantear nuevas tendencias en la modalidad cualitativa, en la que se presta mayor atención a las voces de las personas entrevistadas.

Actualmente se considera que este tipo de entrevista se apoya con fuerza desde la investigación cualitativa, donde el papel de elementos como raza, género, estatus y edad es considerado. Para Suárez (2006), la entrevista es un instrumento de investigación que se sustenta en la técnica de registro de datos, desde la perspectiva comunicacional, por ello Blanchetb y Ghibione (1989, citados en Suárez, 2006) la definen como una conversación entre un entrevistador y un entrevistado, dirigida y registrada por el entrevistador.

Éste tiene por objetivo favorecer la producción de un discurso en una cadena abierta o lineal por parte del entrevistado sobre un tema que se desarrollará en el marco de un trabajo de investigación. Técnica comúnmente usada en el campo de la investigación cualitativa que se preocupa por obtener datos relativos a significados, constructos, experiencias, perspectivas, nociones, conceptos, definiciones, etcétera, en el campo social (Valles, 2014).

\section{Definición de entrevista}

Partiendo de las definiciones de entrevista de Bingham y Moore (1970, citados en Nahoum, 1985), la entrevista es una conversación formal que tiene un objetivo determinado y, a diferencia de una plática informal, nos conduce por un camino planeado donde no se deben perder las riendas de ésta.

Sin embargo, tampoco es un cuestionario aplicado en voz alta; es una interacción bumana que va más allá de recopilar datos e información fría y lejana del entrevistado. Es un encuentro cara a cara que requiere múltiples habilidades profesionales e incluso características personales muy especiales, teniendo como base la empatía de parte del entrevistador.

La mayoría de los autores coinciden en que es un proceso y que, por lo tanto, implica una planeación y una capacitación adecuada para que con el tiempo deje de ser una simple técnica y se convierta en un arte de quien se entrena para ello. Asimismo, tendrá que reconocerse que el tiempo, la experiencia y los errores cometidos brindarán al entrevistador una mirada más bumana y objetiva en sus intervenciones y una especial sensibilidad para conducir a buen puerto la entrevista y al personaje.

La entrevista devela sutilezas del lenguaje verbal y no verbal que no permiten el cuestionario o la encuesta. Tampoco es un análisis de contenido que va en busca de palabras repetidas e interpretaciones semánticas. Es un encuentro de inteligencias y sensibilidades donde la prudencia, la honestidad y la calidez básica dan pie para descubrir actitudes y pensamientos, posturas ante ciertos temas, más que conductas y preguntas y respuestas a 
modo que se podrían adivinar aún antes de iniciar, algo hoy tan común en gran número de periodistas y comunicadores en los medios impresos y electrónicos (Fernández, 1987).

Específicamente en la comunicación y en el periodismo, es frecuente que las entrevistas estructuradas y de perfil no proporcionen mayor confiabilidad y validez que el hecho de que se están cumpliendo los objetivos determinados.

\section{Premisas teóricas:}

\section{la entrevista en la investigación cualitativa}

La entrevista cualitativa permite la recopilación de información detallada en vista de que la persona que informa comparte oralmente con el investigador lo concerniente a un tema específico o evento acaecido en su vida, como lo dicen Fontana y Frey (2005). La misma continúa practicándose mano a mano con el método de la observación participante, aunque también esto es asumido por científicos cuantitativos a quienes les preocupan el rigor de la medición en investigaciones de grandes extensiones.

Así, a los etnógrafos posmodernistas les han preocupado algunas de las asunciones que se presentan en la entrevista y el rol controlador del entrevistador, lo que según Fontana y Frey lleva a que estas preocupaciones se canalicen con nuevas direcciones en la cualitativa, en donde debe focalizarse e incrementarse la atención a las voces de los entrevistados tal y como se mencionó anteriormente.

Rubin y Rubin (1995, citados en Lucca \& Berríos, 2003) enumeran las características que distinguen la entrevista cualitativa de otras formas de recopilar información:

I. La entrevista cualitativa es una extensión de una conversación normal con la diferencia de que uno escucha para entender el sentido de lo que el entrevistador dice.

II. Los entrevistadores cualitativos están inmersos en la comprensión, en el conocimiento y en la percepción del entrevistado, más que en categorizar a personas o eventos en función de teorías académicas.

III. Tanto el contenido de la entrevista cualitativa como el flujo y la selección de los temas cambia de acuerdo con lo que el entrevistado conoce y siente (p. 320).

Se puede decir que la entrevista dentro de la investigación cualitativa es más íntima y flexible. Según Creswell (1997, citado en Hernández, Fernández \& Baptista, 2005), las entrevistas cualitativas deben ser abiertas, sin categorías preestablecidas, de tal forma que los participantes puedan expresar sus experiencias. 
$\mathrm{Al}$ respecto, Alonso (1997) nos indica que:

la entrevista de investigación es por lo tanto una conversación entre dos personas, un entrevistador y un informante, dirigida y registrada por el entrevistador con el propósito de favorecer la producción de un discurso conversacional, continuo y con una cierta línea argumental, no fragmentada, segmentada, precodificado y cerrado por un cuestionario previo del entrevistado sobre un tema definido en el marco de la investigación (p. 228).

\section{Tipos de entrevista}

El tipo de entrevista puede variar de acuerdo con las tácticas que se utilicen para el acercamiento y la situación en la que se desarrolle, por lo que en este artículo se abordarán la entrevista estructurada, la entrevista no estructurada y la entrevista grupal.

En el caso de la entrevista como técnica en la profesión periodística y de los medios de comunicación en general, Leñero y Marín (1996), en su Manual de periodismo, destacan que "es una conversación entre uno o más periodistas con uno o más de un entrevistado" (p.41), y que a través de un diálogo se recolectan diversas noticias, comentarios, opiniones e incluso juicios e interpretaciones de ciertos hechos o eventos, pero que siempre cobra mayor importancia lo que el entrevistado aporte, no lo que el periodista pueda preguntar.

Lo anterior permite la reflexión acerca del protagonismo del comunicador (que no comunicólogo) y del periodista en la mayoría de los medios de comunicación, sobre todo en los electrónicos, donde el hecho de aparecer a cuadro convierte al entrevistador en la estrella y protagonista principal de la historia.

Actualmente, es frecuente observar-sobre todo en la televisión- cómo los conductores de programas como noticiarios, de revista, etcétera, son transformados en la noticia misma, mostrando lo que hacen durante un programa o entrevista y donde el entrevistado pasa a segundo y hasta tercer plano.

Por otra parte, desde Mattelart, pasando por Sartori hasta los teóricos que actualmente estudian la adicción a internet y redes sociales, sostienen-desde diferentes posiciones-que los consumidores y posibles adictos somos los que validamos y legitimamos a estos personajes que hacen de ellos mismos la noticia. Es decir, rendimos culto al periodista, por el cual la nota, reportaje o noticia pasa a segundo y hasta a tercer término. Ya no importa lo que se dice, sino quién lo dijo. Al grado que algunos se han convertido en filósofos que escriben libros de autoayuda de cualquier tema.

Regresando a Leñero, periodista serio, de vocación y oficio incuestionable, la entrevista periodística se divide de la siguiente manera: 
- La entrevista noticiosa o de información

- La entrevista de opinión y

- La entrevista de semblanza.

Por otra parte, Gabriel Bauduco (2008), en su Manual para periodistas, las clasifica en informativas, de declaraciones, de perfiles, de opinión, conferencia de prensa, de ping-pong, encuestas y por asalto.

Finalmente, la entrevista como método o técnica de recolección de datos en la investigación es concebida "como un proceso comunicativo por el cual un investigador extrae una información de una persona” (Alonso, 1997). Para estos autores y siguiendo la lógica de la investigación cualitativa, cobra importancia la palabra, ya que es el vehículo principal de una relación biográfica, única y personalizada.

\section{La entrevista como fenómeno de comunicación}

Entre narradores y escuchas hay una relación directa e imprevisible que es una relación verdaderamente humana... No hablan sólo las palabras, sino los gestos, las expresiones del rostro, los movimientos de las manos, la luz de los ojos. Este es el don de la oralidad: la presencia, el timbre de las voces e incluso el sonido del silencio (Ferrarotti, 1991,p. 19-20 citado en Alonso, 1998).

Sin embargo, es conveniente aclarar que no deberían existir manuales o estándares que conviertan a la entrevista en reglas o mitos derivados de un análisis superficial y carente de sentido científico y metodológico, como las posiciones del cuerpo o los tipos de mirada que deriven en clasificaciones sin fundamento.

\section{Método}

El método utilizado en esta investigación fue de investigación documental de tipo informativa (expositiva).

Esta investigación es básicamente una panorámica acerca de la información relevante de diversas fuentes confiables sobre un tema específico, sin tratar de aprobar u objetar ninguna idea o postura. Toda la información presentada se basa en lo que se ha encontrado en las fuentes. La contribución del investigador radica en analizar y seleccionar de esta información aquello que es relevante para su investigación. Por último, el investigador organiza la información para cubrir todo el tema, sintetizar las ideas y después presentarlas en un reporte final que a la vez sea fluido y esté claramente escrito. 
Asimismo, se hizo un análisis de contenido condensado, documentando el número de ocasiones que se menciona el término entrevista y de conceptos relacionados. También se presenta un breve comentario de cada documento y sus aportaciones al tema de entrevista.

\section{Planteamiento del problema}

¿Existe suficiente y adecuada información bibliográfica, teórica y conceptual acerca de la técnica de la entrevista como herramienta para recopilar información en la investigación?

\section{Objetivos de la investigación}

\section{Objetivo general}

Recopilar y revisar la información bibliográfica, hemerográfica y videográfica que existe acerca de la técnica de entrevista.

\section{Objetivos específicos}

- Revisar las definiciones y modelos teóricos que se han utilizado para desarrollar la técnica de la entrevista.

- Analizar las definiciones, tipos y modalidades de entrevista psicológica.

- Analizar las definiciones, tipos y modalidades de la entrevista de investigación.

- Analizar las definiciones, tipos y modalidades de la entrevista en periodismo y en la comunicación en general.

\section{Metodología}

Se procedió a elegir como fuentes de información documental las siguientes:

- Bibliotecas

- Archivos

- Centros de información hemerográfica y videográfica

- Sitios de internet.

Se utilizaron y consultaron documentos primarios:

1. Libros que tuvieran como tema principal o subtemas a la entrevista como técnica de recolección de información, sin importar su procedencia, enfoque o rama científica 
2. Tesis, investigaciones y memorias que citaran o analizaran los aspectos relacionados con la técnica de entrevista

3. Revistas especializadas y publicaciones periódicas que citaran la entrevista

4. Sitios de internet que documentarán de manera escrita, audiográfica y videográfica aspectos de la entrevista y entrevistas realizadas a diversos personajes de la vida cultural, académica y artística por diferentes tipos de entrevistadores y para varios objetivos.

Los primeros se dividieron en las siguientes categorías:

- Libros, investigaciones y artículos de técnica de entrevista psicológica

- Libros, investigaciones y artículos de entrevista de investigación

- Libros, investigaciones y artículos de entrevista periodística y de la comunicación.

Las referencias de estas fuentes de información analizadas se presentan al final de este artículo.

\section{Definición de investigación documental}

La cantidad de información que se genera en todo el mundo es inmensa. Los pensadores y científicos sobresalientes la incluyen constantemente al acervo del conocimiento mundial. La adquisición de estos conocimientos se hace con frecuencia mediante la investigación documental realizada en fuentes secundarias.

La investigación documental es la presentación de un escrito formal que sigue una metodología reconocida. Esta investigación documental se asigna en cumplimiento del plan de estudios para un curso de preparatoria o de pregrado en la universidad. Consiste primordialmente en la presentación selectiva de lo que expertos ya han dicho o escrito sobre un tema determinado. Además, puede presentar la posible conexión de ideas entre varios autores y las ideas del investigador. Su preparación requiere que éste reúna, interprete, evalúe y reporte datos e ideas en forma imparcial, honesta y clara.

La investigación documental se caracteriza por el empleo predominante de registros gráficos y sonoros como fuentes de información. Generalmente se le identifica con el manejo de mensajes registrados en la forma de manuscritos e impresos, por lo que se le asocia normalmente con la investigación archivística y bibliográfica. El concepto de documento, sin embargo, es más amplio. Cubre, por ejemplo: micropelículas, microfichas, diapositivas, planos, discos, cintas y películas. 


\section{Tipos de investigación documental}

Hay dos tipos de investigación documental: argumentativa e informativa.

Argumentativa (exploratoria). La investigación de este tipo trata de probar que algo es correcto o incorrecto, deseable o indeseable y que requiere solución. Discute consecuencias y soluciones alternas, y llega a una conclusión crítica después de evaluar los datos investigados. Una vez que el tema ha sido seleccionado, el siguiente paso básico es generar preguntas sobre el mismo que puedan guiar la recolección de información significativa al desarrollar la investigación. Existe también el requisito de que el investigador tome partido o determine una postura personal sobre un asunto controvertido, que tratará de apoyar, o probar, con su escrito.

Informativa (expositiva). Este tipo de investigación es básicamente una panorámica acerca de la información relevante de diversas fuentes confiables sobre un tema específico, sin tratar de aprobar u objetar alguna idea o postura. Toda la información presentada se basa en lo que se ha encontrado en las fuentes. La contribución de quien lo realiza radica en analizar y seleccionar de esta información aquello que es relevante para su investigación. Por último, es necesario organizar la información para cubrir todo el tema, sintetizar las ideas y después presentarlas en un reporte final que, a la vez, sea fluido y esté claramente escrito.

Otras formas usuales de investigación son las tesis, que son escritos más extensos basados sobre todo en fuentes primarias y elaboradas como requisito para obtener un título universitario de grado o de posgrado.

\section{Resultados y conclusiones}

En esta investigación se revisaron 25 libros y textos relacionados con el tema de la entrevista, también se encontraron únicamente cinco tesis o investigaciones relacionadas con el tema de la técnica de entrevista y, finalmente, se analizaron 21 documentos videográficos y videos que reseñan diversos estilos de entrevista con personajes varios.

Las siguientes son las conclusiones obtenidas en esta investigación documental:

1. Se encontró que, en los libros y textos de entrevista, tanto el término como la definición de la técnica de la entrevista se cita en muy pocas ocasiones, resaltando las características y habilidades fundamentales del entrevistador y casi sin mención 
a la persona entrevistada. Las diversas posturas acerca de la técnica mencionada varían dependiendo de la orientación, objetivo y formación de los autores y textos revisados. Algunos resaltan la necesidad de seguir una metodología y un proceso muy formal en la conducción y preparación de la entrevista, y otros dan prioridad a la improvisación, dependiendo de la postura y actitudes del entrevistado.

2. En el caso de las tesis e investigaciones se resaltan las cualidades y ventajas de la entrevista sobre otras modalidades de obtención de información en la investigación en las ciencias sociales. Por ejemplo, se desglosan las ventajas de la entrevista abierta, de profundidad y cualitativa, sobre la de tipo estructurada y cerrada.

3. En los videos de diversas entrevistas realizadas a personajes de la ciencia, la cultura y los medios de comunicación se observan diversos estilos de entrevista que permiten concluir que no hay una manera única de conducción, lo cual parece depender de la formación, objetivo y tipo de medio de comunicación con el que está relacionado el entrevistador.

4. En estos últimos se observa que los entrevistados proporcionan más información dependiendo de la postura, actitud y comunicación no verbal den entrevistador, que facilita o inhibe la participación del entrevistado.

5. Finalmente, se puede concluir que la información teórica y conceptual, si bien se encuentra al alcance del investigador, no está unificada y sistematizada de manera que permita un buen desarrollo de la técnica y por consecuencia de las habilidades de un buen entrevistador.

Asimismo, las investigación y ejemplos videográficos proporcionan una amplia gama de estilos que, por una parte, enriquecen el trabajo del entrevistador pero, por otra, no explicitan las cualidades de un buen entrevista dor y en algunas ocasiones se convierten en conversaciones informales con el propósito de hacer sentir cómodo a los entrevistados, pero sin obtener información relevante acerca de éstos o del mismo tema en cuestión.

\section{Referencias}

Acevedo, A. \& López, A. F. (1986). El proceso de la entrevista. Conceptos y modelos. (4ª ed.). México: Limusa.

Alonso, J. A. (1997). La investigación empírica en ciencias sociales. México: Universidad de las Américas.

Alonso, L. (1998) La mirada cualitativa en sociología. Madrid: Editorial Fundamentos. 
Alquímico (mayo 29, 2012). Entrevista a Carlos Monsiváis. 1999. La belleza de pensar. Chile. (Archivo de video). Recuperado de https://www.youtube.com/watch?v=ZtK0iIcVRGU.

Anastasi, A. (1998). Los tests psicológicos. (7ª ed.). México: Pearson Educación.

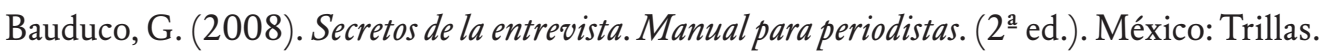

Barthes, R. (1983). El grano de la voz: Entrevistas 1962-I980. México: Siglo XXI.

Bastenier, M. A. (2014). La entrevista como reportaje I y II. El País. Recuperado de http://cultura.elpais.com/cultura/2014/12/19/actualidad/1419018971_886196.html.

Báez, A. (mayo 7, 2014). Salvador Dalí muy mal entrevistado por Facobo Zabludowsky. (Archivo de video). Recuperado de https://www.youtube.com/watch?v=fQBcP7nxsDc.

Canal 11 (octubre 1, 2012). Entrevista a Facobo Zabludowsky en el programa Conversando con Cristina Pacheco. (Archivo de video). Recuperado de https://www.youtube.com/watch?v=boiSwFC0vLA.

Canal de Aragón (enero 24, 2010). Silvia Lemus entrevista a Miguel Ríos en el programa "Tratos y retratos". (Archivo de video). Recuperado de YouTube: https://www.youtube.com/watch?v=eXBhubvGRzE.

Canal Oficial Extranormal (enero 19, 2011). Favier Alatorre entrevista a Fosé Forge Balderas “77”. (Archivo de video). Recuperado de https://www.youtube.com/watch?v=HBCqPi61 pow.

Capote, T. (1968). A sangre fría. Barcelona: Editorial Noguer.

Camposeco, R. (marzo 13, 2015). Noticieros Televisa con Foaquín López Dóriga: Valentina Alazraki entrevista al Papa Francisco. Dospartes. (Archivo de video). Recuperado de https://www.youtube. com/watch?v=32qbZU0ahg0.

Confidencial (mayo 11, 2014). Entrevista a Fuan Villoro en programa Confidencial. "Dios es redondo. Sobre futbol, literatura y periodismo". (Archivo de video). Recuperado de https://www.youtube. com/watch?v=4Xn1zYmksrE.

Cousseau, C. (junio 7, 2013). Foaquín Soler Serrano entrevista a Forge Luis Borges en programa A Fondo, 1976. (Archivo de video). Recuperado de https://www.youtube.com/watch?v=2gu91_TqS8I.

Cheestoslandia (octubre 24, 2014). Entrevista a Fosé Mujica (completa). En CNN Aristegui (Archivo de video). Recuperado de https://www.youtube.com/watch?v=j1zDRjrsxS0.

CNN Chile (noviembre 22, 2011). Daniel Mosciatti entrevista a Camila Vallejo. Dirigente estudiantil de Chile en el programa de radio Bio-Bio. (Archivo de video). Recuperado de https://www. youtube.com/watch?v=vcJLDfQnndA.

Davis, F. \& Newstron, R. (1987). El comportamiento bumano en el trabajo. Nueva York: McGraw-Hill.

Delfinespro (diciembre 13, 2011). Redes (№ 114)-De las inteligencias múltiples a la educación personalizada. Entrevista de Eduard Punset a Howard Gardner, al recibir el Premio Príncipe de Asturias en las Ciencias Sociales 20II. (Archivo de video). Recuperado de https://www.youtube.com/watch?v=DUJL1V0ki38.

Denzin N. \& Lincoln, Y. (2005). The Sage Handbook of Qualitative Research. Third Edition. New York: Thousand Oaks Sage Publications. Pp. 1-13.

Díaz, I. (2008). Técnica de la entrevista psicodinámica. México: Editorial Pax. 
Díaz, L., Torruco, U., Martínez, M. \& Varela, M. (2013). La entrevista, recurso flexible y dinámico. Investigación en Educación Médica, 2(7), 162-167. Recuperado de http://riem.facmed.unam.mx/ sites/all/archivos/V2Num03/09_MI_LA\%20_ENTREVISTA.pdf

Dorfman, A. \& Mattelart, A. (1983). Para leer al Pato Donald. Comunicación de masa y colonialismo. México: Siglo XXI.

Eduardo, A. (abril 30, 2015). La entrevista por Adela. Joaquín Sabina. (Archivo de video). Recuperado de https://www.youtube.com/watch?v=NCyI_1QUdNE.

Fallaci, O. (1980). Entrevista con la bistoria. Colombia: Círculo de Lectores.

Fernández, P. (1987). La entrevista. México: Asociación Mexicana de Profesionales de la Orientación.

Fontana, A. \& Frey, J. (2005). The Interview, from neutral stance to political involvement. En N. K. Denzin \& y S., Lincoln (Comp.). The Sage Handbook of Qualitative Research (695-727). London: Sage.

Franco, I. (2013). Tesis de investigación. Entrevistas. En Y. Franco, Tesis de investigación [Blog de internet].Venezuela.Franco Yaquelin.Disponibleenhttp://tesisdeinvestig.blogspot.com/2011/06/ entrevistas.html.

García, G. (2000). El olor de la guayaba. México: Editorial Diana.

Gubern, R. (1996). Del bisonte a la realidad virtual. Barcelona: Anagrama.

Halperín, J. L. (1995). La entrevista periodística: Intimidades de la conversación pública. Buenos Aires: Paidós.

Hernández, R., Fernández, C. \& Baptista P. (2010). Metodología de la investigación. (5ํaed.). México: McGraw-Hill.

Hernández, M., García, S., López, N. \& Rodríguez, M. (1 de diciembre de 2015). Estudio de encuestas. Recuperado de https://www.uam.es/personal_pdi/stmaria/jmurillo/InvestigacionEE/Presentaciones/Curso_10/ENCUESTA_Trabajo.pdf.

Herrera, M. (abril 29, 2012). Foaquín Soler Serrano entrevista a Fuan Rulfo. (Archivo de video). Recuperado de https://www.youtube.com/watch?v=V74yJztkx-c.

Lemus, S. (2015). La entrevista debe ser un monólogo provocado. La Fornada. Recuperado de http://www.jornada.unam.mx/2014/03/03/cultura/a08n1 cul.

Leñero, V. \& Marín, C. (1996). Manual de periodismo. México: Grijalbo.

López, A. (abril 28, 2015,). Entrevista a AM L O con Facobo Zabludowsky. (Archivo de video). Recuperado de https://www.youtube.com/watch?v=yMTqQrYTdP8.

López, R. \& Dealuriers, J. P. (2011). La entrevista cualitativa como técnica para la investigación. Revista Margen, No. 61. Recuperado de http://www.margen.org/suscri/margen61/lopez.

Lucca, N. \& Berríos, R. (2003). La investigación cualitativa. fundamentos, diseños y estrategias. Colombia: Ediciones S.M. Recuperado de https://dialnet.unirioja.es/descarga/articulo/3945773.pdf

Knapp, M. L. (1978). Non Verbal Communication in Human Interaction. New York: Holt, Rinehart y Winston. 
Kvale, S. (1996). Interviews. An Introduction to Qualitative Research Interviewing. Thounsand Oaks, London: Sage Publications.

Lipton, J. (febrero 11, 2015). Fames Lipton entrevista a Brad Pitt en el Programa Inside The Actors Studio. (Archivo de video). Recuperado de YouTube: https://www.youtube.com/watch?v=cFfarLUWjpo.

Mattelart, A. \& Mattelart, M. (1994). Los medios de comunicación en tiempos de crisis. México: Siglo XXI.

Martínez, P. (2001). Manual básico de investigación científica. México: Manual Moderno/Universidad Anáhuac México.

Morga, L. E. (2012). Teoría y técnica de la entrevista. México: Editorial Red Tercer Milenio.

Muñoz, C. (2008). Como elaborar y asesorar una investigación de tesis. México: Pearson Educación.

Nahoum, Ch. (1985). La entrevista psicológica. México: Kapelusz.

Peláez, A. \& Rodríguez, J. Artículo de investigación de entrevista. Recuperado de https://www.uam.es/ personal_pdi/stmaria/jmurillo/InvestigacionEE/Presentaciones/Curso_10/Entrevista_trabajo.pdf.

Prudencio, A. (agosto 12, 2012,). Entrevista a Gabriel García Márquez tras el Nobel de Literatura. (Archivo de video) Recuperado de https://www.youtube.com/watch?v=XF9J1PEOY8U.

Restrepo, E. La entrevista como técnica de investigación social. Instituto de Investigaciones. Recuperado de https://www.ramwan.net/restrepo/documentos/entrevista.

Romelaer, P. (2009). Entrevistas como medios para recolectar datos científicos. Universidad París Dauphine. Recuperado de http://aeo.izt.uam.mx/seminario/tendencias/La-entrevista.pdf.

Rubin, H. J. \& Rubin, I. S. (1995). Qualitative Interviewing. Thousand Oaks, CA: Sage.

Rueda, A. (2008). De la mirada al texto: la entrevista literaria de Elena Poniatwska. Boletín de la Unidad de Investigación de la Universidad de Alicante. ISSN 1577-3442, №. 11-12. Recuperado de http://dialnet.unirioja.es/servlet/revista?codigo $=2779$.

Sánchez de Armas, M. A. (1998). Apuntes para una bistoria de la televisión mexicana. México: Revista Mexicana de Comunicación/Televisa..

Sandín, M. P. (2003). Investigación cualitativa en educación. México: McGraw-Hill.

Santoro, D. (2004). Técnicas de investigación. Métodos desarrollados en diarios y revistas de América Latina. México: Fondo de Cultura Económica.

Sartori, G. (1998). Homo Videns. La sociedad teledirigida. Madrid: Taurus.

Silvester, Ch. (1997). Las grandes entrevistas de la bistoria. México: El País/Aguilar.

Solís, E. (enero 19, 2011). Entrevista de Carlos Loret de Mola a Fosé Forge Balderas Garza el "F7". (Archivo de video). Recuperado de https://www.youtube.com/watch?v=9LfTZFukhW8.

Suárez, M. (2006). Técnicas e instrumentos para la recolección de la información. Universidad Rovira I Virgili. Tarragona, Cataluña, España. Recuperado de http://www.tesisenred.net/bitstream/ handle/10803/8922/42CapituloIVMarcoReferencialmetodologicotfc2.pdf?sequence=9.

Valles, M. (2014). Técnicas cualitativas en investigación social. Madrid: Síntesis.

Vargas, I. (2011). La entrevista en la investigación cualitativa: nuevas tendencias y retos. Revista CAES, Vol. 3I, No. 1, 122.

Vidal, D. (1998). La entrevista en radio, televisión y prensa. Madrid: Cátedra. 